\title{
Analysis on Gel Electrophoresis in Biology
}

\author{
Yiran Cai \\ Hangzhou Foreign Languages School, 299 LiuHe Road, Hangzhou, Zhejiang Province, 310023, P. R. China
}

\begin{abstract}
Gel electrophoresis is one of the basic methods used in modern biological laboratories to separate different compounds in a mixture. It is widely used in analyzing the amino acid sequence in proteins. In the following sections, we will discuss the principles of gel electrophoresis and the current challenges of this technology. Gel electrophoresis is used based on the size, mass, and charge of each particle while the movement of molecules depends on the potential differences in power supply and the channel through which the gel is formed. The composition, properties and indexes of the gel are introduced. The gels, nevertheless, have flaws for scientific studies. In this paper, the mechanism of gel electrophoresis and current challenges confronting it will be discussed as references for further improvement of this technology.
\end{abstract}

\section{Introduction}

Electrophoresis is a technology used to separate molecules such as amino acid from DNA. In this process, those macromolecules are separated by their mass, charge and size. In addition, the channels inside of the gels forms the path in which the particles travel regularly. Through these gels, particles in smaller mass and size can move faster, whereas the larger ones move relatively slowly. Based on the above properties, electrophoresis has been widely applied in various fields such as DNA sequencing and fingerprinting.

However, though still unknown, the electrophoresis also has drawbacks. For instance, agarose gel electrophoresis, the dominant technique in modern laboratory for DNA sequencing and recycling, has limited resolution ratio for macromolecules, making it difficult to obtain clear DNA strip images. Moreover, the deformation of sample mixture and changing experimental conditions are vital issues demanding further considerations.

Therefore, the main purpose of this paper aims at illustrating the basic principle of gel electrophoresis, modernist composition as well as applications in the modern world. The paper also focuses on the flaws of gel electrophoresis to propose potential solutions in this regard.

\section{Method and Analysis}

\subsection{Method to Carry Out Gel Electrophoresis}

The protocol for this technology consists of a three-step process: preparing gels and other materials, running a power-on electrophoresis, and placing the gel under ultraviolet for observation.

\subsubsection{Material preparing}

The main gels and buffer are the most important materials for this experiment, which could be divided into two types of components.

Agarose gel

$50 \mathrm{mM}$ Tris-base, $30 \mathrm{mM}$ sodium acetate, $3 \mathrm{mM}$ EDTA in deionized water, approximate $\mathrm{pH} 7.8$ with $96 \%$ acetic acid.

TEA buffer: $2 \mathrm{M}$ sucrose, $100 \mathrm{mM}$ EDTA, $0.2 \mathrm{mM}$ bromophenol blue.

\section{Indicator}

As gels and macromolecules are typically colorless and transparent, the original strips on the gel are invisible after being exposed to ultraviolet. Thus, their presence is identified either by staining or by using gene probes.

Gene probes are single-stranded DNA with a base sequence complementary to that of the targeting fragment or gene. The probe must be radioactive so that when the treated gel is exposed to X-ray film, the presence of that specificprobe and the fragment attached to it will be revealed. Alternatively, the probe must have a fluorescent staining attachment that glows distinctively under ultraviolet light, thereby revealing the presence of the traget fragment or gene.

The other materials include a chamber for loading gels and buffer, power supply, and pipettes.

Besides, in some labs, the gels are made of polyacrylamide with TEB buffer. 


\subsubsection{Procedure and result}

1. Place the agarose gel in the electrophoresis apparatus, fill the electrophoresis chamber with $1 \mathrm{x}$ TAE buffer to cover the gel.

2. Use a separate nozzle for each sample, and insert the sample into the well.

3. Place the lid and the switch on $100 \mathrm{~V}$ power supply and electrify for $30 \mathrm{~min}$.

4. Expose the gel to ultraviolet $(254 \mathrm{~nm})$ and collect the images.

\section{Result}

Different bands of gel are visible under the ultraviolet light, representing different DNA fragments. The same position indicates the same DNA fragments as molecules of the same type move at equal speed.

\subsection{Different materials' properties}

In the practical experiment, different gels and buffer aim to match various traits of the samples.

\subsubsection{Agarose gel and polyacrylamide gel}

Although both agarose and polyacrylamide gels are widely used in biochemistry lab, they have different aims due to their specific properties.

Agarose gel is the simplest electrophoresis because it's easy to prepare. Also, agarose gel electrophoresis can be easily performed because the sample does not require special treatment. Moreover, the uniform inner structure and a high degree of moisture of agarose gel provide ideal electrophoresis conditions (almost free electrophoresis). Besides, the transparency of agarose gel allows ultraviolet to passes through it freely, resulting in direct images [1]. However, agarose gels have a lower resolution ratio than polyacrylamide gel, making it the dominant technique to separate large molecules such as virus and big nucleic acid.

Polyacrylamide gel is another gel used in biology lab. Compared with agarose gel, polyacrylamide gel is an artificial gel, whose polymer property allows its recycling. The polyacrylamide gel can better separate the macromolecules than agarose gel. Also, polyacrylamide can be adjusted to change the size of the channel in order to accurately separate molecules. Nonetheless, because $\mathrm{pH}$ is a significant factor in polyacrylamide gel electrophoresis, the change of ion concentration can easily lead to the change of electrophoresis result.

\subsubsection{TAE buffer and TBE buffer}

Buffer is the liquid that regulates the $\mathrm{pH}$ of electrophoresis. The electricity flows in the liquid could cause redox reaction, thus changing the ionic concentration in the liquid and affecting the reaction process or even the result of the experiment. Therefore, buffer is used to balance the $\mathrm{pH}$ at each pole, helping molecules to migrate smoothly.

TAE buffer is the most commonly used buffer in electrophoresis as it could better separate the DNA spiral structure. When there is the need to recycle DNA, TAE buffer is the most appropriate choice. TBE buffer is used for electrophoresis with a long duration, such as overnight electrophoresis [2].

\section{Discussion}

\subsection{Modern applications of DNA electrophoresis}

Gel electrophoresis is mostly widely used in biology and biochemistry laboratory to examine dyes, amino acid and DNA fragments. Besides, gel electrophoresis can also be used for forensic purposes.

\subsubsection{DNA finger printing}

It is widely known that every person has unique DNA, similar to fingerprint. Therefore, this cutting-edge technology can be used in personal identification and paternity test.

The procedures of the process is simple. First, the DNA should be extracted from the creature sample, where the DNA molecules would be amplified though polymerase chain reaction to obtain explicit results. Then, electrophoresis is used to separate the DNA molecules based on their molecular mass. After manipulating the DNA strips, the DNA fingerprint can be used to determine the person's identification.

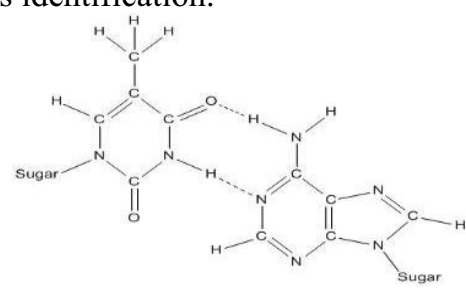

Figure 1 The diagram shows the structure of DNA molecule.

*The hydroxyl group in phosphate is dissociated, and therefore the DNA molecule is always negatively charged. Thus, the DNA molecule moves to the positive side of the electric field.

\subsubsection{Checking PCR}

PCR (polymerase chain reaction) is a technology to amplify the DNA molecules, like natural DNA replication. In order to determine whether the replication is completely consistent with the original sample, gel electrophoresis is utilized for this purpose. Typically, fluorescent staining serves as the indicator to visualize each DNA strip under the ultraviolet. With the technological development, fluorescent has become the most popular method.

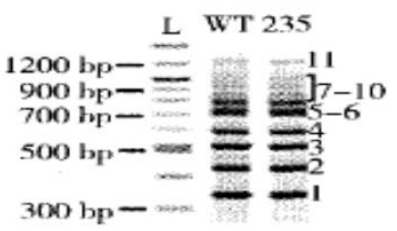

Figure 2 The gel image of PCR result under the u.v light. 
'Bp' represents the number of base pairs of each sample. The number of base pairs decreases as the distance from the origin increases.

\subsection{Current challenges for gel electrophoresis}

Nevertheless, because the gel electrophoresis is carried out in highly dynamic liquid, the results could be affected by multiple factors, including the quality of image under the ultraviolet, recycling of DNA as well as protein examination. The causes of the above problems will be explained in detail.

When the electricity flows through the liquid, the redox reaction takes place at two electrodes, which would form the hydrogen ions and hydroxide ions. However, this situation could seriously disrupt the $\mathrm{pH}$ condition of electrophoresis as the gel electrophoresis generally run under $\mathrm{pH}$ 8.3. Adverse changes in $\mathrm{pH}$ could make it difficult to perform the experiment.

The shape of the channel in agarose gel for electrophoresis is fixed. In contrast, the molecules of DNA or proteins have a variety of appearance. Therefore, not all the molecules can easily pass through the channel. In some cases, researchers can use this theory to filter out parts of the molecules with unique shapes. However, the gels bear certain limitations. For example, the linearly branched polymers prefer channel structures while globular molecules travel more efficiently in Brownian rectifiers [3]. To optimize the efficiency of the gel electrophoresis, it is necessary to solve the problems caused by channel shapes.

The third contributing factor lies in deformation of molecules caused by electric filed intensity. Obviously, the DNA molecules and proteins have polar structure, which is formed by hydrogen bond and ionic bond. However, the intensity of electric filed is a vital factor weakening those bonds or even breaking down the whole structure [4], thus preventing us from obtaining precise results.

The medium for electrophoresis can also be made up of polymer mixture. The polymer mixture, however, has problems as well as it could entangle with the molecules transiently, drastically affecting the electrophoresis process. The polyelectrolyte velocity goes to a minimum when the contour length of the polymer is varied [5]. In other words, the range of molecular sizes for test is dramatically restricted. Thus, optimizing the range of the polymer mixture is also essential.

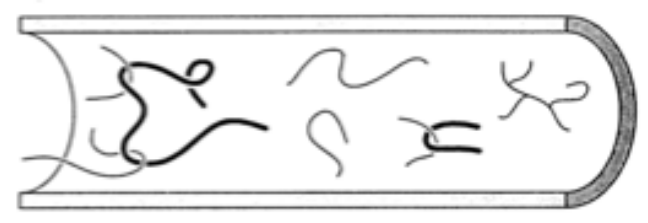

Figure 3 Collisions between long DNA molecules and the polymer chains of solution provide very fast separations.

Some of the long DNA molecules are isolated in the pulsed fields instead of in the steady fields. Therefore, DNA molecules and polymers are electrically excited, producing effective results. The size range of the DNA could be expanded along with increases in the intensity of the filed [6]. Also, maximum extension of DNA is dependent upon the frequency and intensity of the electric field [7]. However, how to excite the DNA molecules and polymer dynamical mode to the optimal level is still unknown.

\subsection{Suggestions and improvement}

To improve the quality of the electrophoresis result, the first problem demanding a solution is the $\mathrm{pH}$ condition. Changes in the $\mathrm{pH}$ makes it impossible for long-duration electrophoresis. Therefore, the buffer's resistance should be emphasized and improved. The proportion of weakbased buffer should be optimized to accommodate the maximum sample size.

The highly specialized shape of the gel's channels is another issue for consideration. In order to maximize the efficiency of the electrophoresis, a more flexible gel should be developed to suit the individual needs of several mixture samples simultaneously.

As to the ionic bond damage and medium entanglement, the aided reagent can be applied to prevent the deformation by providing appropriate environment to maintain a stable ionic bond structure and measuring range during the whole experiment.

To testify the optimum electric field intensity, more experiments are needed to find out the most suitable electrophoresis condition, including gel thickness, electric intensity and temperature.

\section{Conclusion}

Gel electrophoresis is used prevalently in laboratory to separate various compounds in mixture for further analysis of amino acid and DNA recycling. Also, it is utilized in forensic to determine personal identity. However, this technology is still confronted with challenges, which demands further improvement to bring more benefits to the scientific researches. This paper presents an overview of problems in gel electrophoresis coupled with possible solutions to perfect this technology, which serve as references for future studies. Subsequent researches will mainly focus on exploring new buffer, liquid medium, and optimum conditions for gel electrophoresis.

\section{References}

1. J. H. Xu, and P. C. Dedon, "Technologies for Detection of DNA Damage and Mutations. Edited by Gerd P. Pfeifer." Photochemistry and Photobiology 65, 3, pp.603-604 (1997)

2. M. Dybiec, et al, "Photoluminescence spectroscopy of bioconjugated $\mathrm{CdSe} / \mathrm{ZnS}$ quantum dots." Applied Physics Letters 90, 26, p.9463 (2007)

3. T. A. J. Duke, and R. H. Austin, "Microfabricated Sieve for the Continuous Sorting of Macromolecules." Physical Review Letters 80, 7, pp.1552-1555 (1998) 
4. B. E. Annelise, D. S. Soane, and H. W. Blanch, "Capillary Electrophoresis of DNA in Uncrosslinked Polymer Solutions." Journal of Chromatography A 652, 1, pp.3-16 (1993)

5. C. Desruisseaux, G. W. Slater, and T. B. L. Kist, "Trapping Electrophoresis and Ratchets: A Theoretical Study for DNA-Protein Complexes." Biophysical Journal 75, 3, pp.12281236 (1998)

6. S. F. Edwards, and M. Muthukumar, "The size of a polymer in random media." The Journal of Chemical Physics 89, 4, pp.2435-2441(1988)

7. M. Muthukumar, and A. Baumgaertner, "Diffusion of a polymer chain in random media." Macromolecules 22, 4, pp.1941-1946(1989) 